\title{
Sums over primes II
}

\author{
Rusen Li \\ School of Mathematics \\ Shandong University \\ Jinan 250100 China \\ limanjiashe@163.com
}

2020 MR Subject Classifications: 11B83, 11L20, 11N25, 11N37

\begin{abstract}
In this paper, we give explicit asymptotic formulas for some sums over primes involving generalized alternating hyperharmonic numbers of types I, II and III. Analogous results for numbers with $k$-prime factors will also be considered.

Keywords: sums over primes, generalized alternating hyperharmonic numbers, asymptotic formula, numbers with $k$-prime factors
\end{abstract}

\section{Introduction and preliminaries}

It is known to all that prime numbers play an essential role in number theory. The infamous problem (known as the Prime Number Theorem) that how many primes there are up to a given point has attracted many excellent mathematicians' interests since the time of Euclid. Let $\pi(x)$ denote the number of primes up to $x$. Gauss and Legendre proposed independently that the ratio $\pi(x) / \frac{x}{\log x}$ would approach 1 as $x$ approaches $\infty$. With the help of analytic tools, Hadamard [6] and de la Vallée Poussin [2] independently and almost simultaneously proved the Prime Number Theorem, i.e.,

$$
\pi(x) \sim \frac{x}{\log x} .
$$

Here, and through out this paper, we use the natural logarithm (to base $e)$. We write $A(x) \sim B(x)$, that is $A(x)$ is asymptotic to $B(x)$, which is equivalent to

$$
\lim _{x \rightarrow \infty} \frac{A(x)}{B(x)}=1
$$


Let $p_{n}$ be the sequence of prime numbers, the Prime Number Theorem can be restated as

$$
\pi(x)=\sum_{p_{n} \leq x} p_{n}^{0} \sim \frac{x}{\log x} .
$$

It is natural to consider asymptotic formulas for more general sums of type $\sum_{p_{n} \leq x} p_{n}^{\alpha}$. An exercise in Granville's book [5] states that $\sum_{p \leq x} p \sim \frac{x^{2}}{2 \log x}$. This result can be proved with the help of asymptotic formula of $p_{n}$, which is equivalent to the Prime Number Theorem. In fact, we can prove asymptotic formulas for $\sum_{p_{n} \leq x} p_{n}^{\alpha}$, i.e., $\sum_{p_{n} \leq x} p_{n}^{\alpha} \sim \frac{x^{1+\alpha}}{(1+\alpha) \log x}$, which is first obtained by Šalát and Znám [16]. Later, Jakimczuk [8, 9] extends this kind of summation to numbers with $k$ prime factors and functions of slow increase. Gerard and Washington [4] also give accurate estimates for $\sum_{p_{n} \leq x} p_{n}^{\alpha}-\frac{x^{1+\alpha}}{(1+\alpha) \log x}$ by using the Prime Number Theorem with error terms.

The above results remind the author that it would be interesting to obtain asymptotic formulas for sums over primes of types $\sum_{p_{n} \leq x} p_{n}^{\alpha} f(n)^{m}$, where $f(n)$ denotes an arithmetical function. Motivated by an exercise in Granville's book [5] and the author's recent work [12] on generalized hyperharmonic numbers $H_{n}^{(p, r)}$, the author [13] give explicit asymptotic formulas for sums over primes involving generalized hyperharmonic numbers of type $\sum_{p_{n} \leq x} p_{n}^{\alpha}\left(H_{n}^{(p, r)}\right)^{m}$. Analogous results for numbers with $k$-prime factors have also be considered by the author [13].

We now recall the definition of numbers with $k$-prime factors and the hyperharmonic numbers. Let $k \geq 1$ and consider a positive integer $n$ which is the product of just $k$ prime factors, i.e.,

$$
n=p_{1} p_{2} \cdots p_{k}
$$

We write $\tau_{k}(x)$ for the number of such $n \leq x$. If we impose the additional restriction that all the prime divisors $p$ in (3) shall be different, $n$ is squarefree. We write $\pi_{k}(x)$ for the number of these (squarefree) $n \leq x$. It was proved by Landau [7, 10] that

$$
\pi_{k}(x) \sim \tau_{k}(x) \sim \frac{x(\log \log x)^{k-1}}{(k-1) ! \log x} \quad(k \geq 2) .
$$

For $k=1$, this result would reduce to the Prime Number Theorem, if, as usual, we take $0 !=1$. 
The conception of hyperharmonic numbers are first introduced by Conway and Guy in their famous book [1] as

$$
h_{n}^{(r)}:=\sum_{j=1}^{n} h_{j}^{(r-1)} \quad(n, r \in \mathbb{N}:=\{1,2,3, \cdots\}) \quad \text { with } \quad h_{n}^{(1)}=H_{n}:=\sum_{j=1}^{n} 1 / j .
$$

From the definition of hyperharmonic numbers we can see that these numbers can be obtained by taking repeated partial sums of harmonic numbers $H_{n}$. Starting from the classical generalized harmonic numbers $H_{n}^{(p, 1)}=$ $H_{n}^{(p)}:=\sum_{j=1}^{n} 1 / j^{p}$ and taking repeated partial sums, Dil, Mező and Cenkci [3] introduced the generalized hyperharmonic numbers

$$
H_{n}^{(p, r)}:=\sum_{j=1}^{n} H_{j}^{(p, r-1)} \quad(n, p, r \in \mathbb{N}),
$$

and studied their Euler sum properties. Ömür and Koparal [15] introduced the generalized hyperharmonic numbers $H_{n}^{(p, r)}$ independently and almost simultaneously from a combinatorial point of view, and defined two $n \times n$ matrices $A_{n}$ and $B_{n}$ with $a_{i, j}=H_{i}^{(j, r)}$ and $b_{i, j}=H_{i}^{(p, j)}$, respectively. They also gave some interesting factorizations and determinant properties of the matrices $A_{n}$ and $B_{n}$. The author [12] proved that the generalized hyperharmonic numbers $H_{n}^{(p, r)}$ could be written in terms of linear combinations of $n$ 's power times generalized harmonic numbers.

The conception of generalized alternating hyperharmonic numbers are introduced by the author [1] as an alternating analogue of the generalized hyperharmonic numbers $H_{n}^{(p, r)}$. Define the notion of the generalized alternating hyperharmonic numbers of types I, II, and III, respectively, as

$$
\begin{aligned}
H_{n}^{(p, r, 1)} & :=\sum_{k=1}^{n}(-1)^{k-1} H_{k}^{(p, r-1,1)} \quad\left(H_{n}^{(p, 1,1)}=H_{n}^{(p)}\right), \\
H_{n}^{(p, r, 2)} & :=\sum_{k=1}^{n} H_{k}^{(p, r-1,2)} \quad\left(H_{n}^{(p, 1,2)}=\bar{H}_{n}^{(p)}:=\sum_{j=1}^{n}(-1)^{j-1} / j^{p}\right), \\
H_{n}^{(p, r, 3)} & :=\sum_{k=1}^{n}(-1)^{k-1} H_{k}^{(p, r-1,3)} \quad\left(H_{n}^{(p, 1,3)}=\bar{H}_{n}^{(p)}\right) .
\end{aligned}
$$

Let $\mathbb{N}_{0}$ denote the set of nonnegative integers. If $p \in \mathbb{N}_{0}$, then $H_{n}^{(-p)}$ and $\bar{H}_{n}^{(-p)}$ are understood to be the sum $\sum_{j=1}^{n} j^{p}$ and $\sum_{j=1}^{n}(-1)^{j-1} j^{p}$, respectively. The author [11] proved that Euler sums of the generalized alternating 
hyperharmonic numbers of types I, II, and III could be expressed in terms of linear combinations of classical (alternating) Euler sums.

The motivation of this paper arises from an exercise in Granville's book [5] and the author's recent work [11] on generalized alternating hyperharmonic numbers of types I, II and III. This paper is a continuation of the previous paper of the author with the same title [13]. In this paper, we will derive explicit asymptotic formulas for some sums over primes involving generalized alternating hyperharmonic numbers of types I, II and III. Analogous results for numbers with $k$-prime factors will also be considered.

\section{Some notations and lemmata}

We now recall some notations and lemmata which will be useful in later sections.

Lemma 1 ([14]). For all $n \in \mathbb{N}$ and a fixed order $r \geq 1$, we have

$$
h_{n}^{(r)} \sim \frac{1}{(r-1) !} n^{r-1} \log (n) .
$$

Lemma 2 ([13]). For $r, n, p \in \mathbb{N}$ with $p \geq 2$, we have

$$
H_{n}^{(p, r)} \sim \frac{1}{(r-1) !} n^{r-1} \zeta(p),
$$

where $\zeta(p):=\sum_{n=1}^{\infty} n^{-p}$ denotes the well-known Riemann zeta function.

Lemma 3 ([12]). For $r, n, p \in \mathbb{N}$, we have

$$
H_{n}^{(p, r, 2)}=\sum_{m=0}^{r-1} \sum_{j=0}^{r-1-m} a(r, m, j) n^{j} \bar{H}_{n}^{(p-m)} .
$$

The coefficients $a(r, m, j)$ satisfy the following recurrence relations:

$$
\begin{aligned}
a(r+1, r, 0)= & -\sum_{m=0}^{r-1} a(r, m, r-m-1) \frac{1}{r-m}, \\
a(r+1, m, \ell)= & \sum_{j=\ell-1}^{r-1-m} \frac{a(r, m, j)}{j+1}\left(\begin{array}{c}
j+1 \\
j-\ell+1
\end{array}\right) B_{j-\ell+1}^{+} \\
& (0 \leq m \leq r-1,1 \leq \ell \leq r-m),
\end{aligned}
$$


$a(r+1, m, 0)=-\sum_{y=0}^{m} \sum_{j=\max \{0, m-y-1\}}^{r-1-y} a(r, y, j) D(r, m, j, y) \quad(0 \leq m \leq r-1)$,

where

$$
D(r, m, j, y)=\sum_{\ell=\max \{0, m-y-1\}}^{j} \frac{1}{j+1}\left(\begin{array}{c}
j+1 \\
j-\ell
\end{array}\right) B_{j-\ell}^{+}\left(\begin{array}{c}
\ell+1 \\
m-y
\end{array}\right)(-1)^{1+\ell-m+y}
$$

and Bernoulli numbers $B_{n}^{+}$are determined by the recurrence formula

$$
\sum_{j=0}^{k}\left(\begin{array}{c}
k+1 \\
j
\end{array}\right) B_{j}^{+}=k+1 \quad(k \geq 0)
$$

or by the generating function

$$
\frac{t}{1-e^{-t}}=\sum_{n=0}^{\infty} B_{n}^{+} \frac{t^{n}}{n !}
$$

The initial value is given by $a(1,0,0)=1$.

Definition 1. For $m, j \in \mathbb{N}_{0}$, define the quantities $c(m, j), d(m, j), c_{1}(m, j)$, and $d_{1}(m, j)$ as

$$
\begin{aligned}
& c(m, j)=\frac{1}{m+1}\left(\begin{array}{c}
m+1 \\
m+1-j
\end{array}\right) B_{m+1-j}^{+}, \\
& d(m, j)=\frac{1}{m+1} \sum_{k=j-1}^{m}\left(\begin{array}{c}
m+1 \\
m-k
\end{array}\right) B_{m-k}^{+}\left(\begin{array}{c}
1+k \\
j
\end{array}\right)(-1)^{1+k-j}, \\
& c_{1}(m, j)=\frac{1}{2(m+1)} \sum_{k=0}^{m-j}\left(\begin{array}{c}
m+1 \\
k
\end{array}\right) B_{k}^{+} 2^{k}\left(\begin{array}{c}
m+1-k \\
j
\end{array}\right)(-1)^{m-k-j}, \\
& d_{1}(m, j)=\sum_{k=j}^{m}\left(\begin{array}{c}
k \\
j
\end{array}\right)(-1)^{k-j} c_{1}(m, k) .
\end{aligned}
$$

Definition 2. For $r \in \mathbb{N}$, define the quantities $b_{1}(r, m, j, k), k=0,1,2,3$ as

$$
\begin{aligned}
& b_{1}(1,0,0,2)=1, \quad b_{1}(1,0,0,3)=0, \quad b_{1}(r, m, j, 0)=b_{1}(r, m, j, 1)=0 \quad(r=\text { odd }), \\
& b_{1}(r, m, j, 2)=b_{1}(r, m, j, 3)=0 \quad(r=\text { even }), \\
& b_{1}(r, m, j, 3)=0 \quad\left(r=\text { odd }, \quad m+j=\frac{2 r-(-1)^{r}-3}{4}\right),
\end{aligned}
$$


and for $k=0,1,2,3, b_{1}(r, m, j, k)$ satisfy the following recurrence relations: when $r$ is odd,

$$
\begin{aligned}
& b_{1}(r+1, m, j, 0)=\sum_{\ell=m}^{\frac{2 r-(-1)^{r}-3}{4}} b_{1}(r, \ell, j, 2) c_{1}(\ell, m), \\
& \left(1 \leq m \leq \frac{2 r-(-1)^{r}-3}{4}, \quad 0 \leq j \leq \frac{2 r-(-1)^{r}-3}{4}-m\right) ; \\
& b_{1}(r+1,0, j, 0)=\sum_{\ell=0}^{\frac{2 r-(-1)^{r}-3}{4}-j} b_{1}(r, \ell, j, 2) c_{1}(\ell, 0),\left(0 \leq j \leq \frac{2 r-(-1)^{r}-3}{4}\right) \text {; } \\
& b_{1}(r+1, m, j, 1)=\sum_{\ell=m-1}^{\frac{2 r-(-1)^{r}-3}{4}-1} b_{1}(r, \ell, j, 3) c(\ell, m) \text {, } \\
& \left(1 \leq m \leq \frac{2 r-(-1)^{r}-3}{4}, \quad 0 \leq j \leq \frac{2 r-(-1)^{r}-3}{4}-m\right) \text {; } \\
& b_{1}(r+1,0, j, 1)=\sum_{m=0}^{\frac{2 r-(-1)^{r}-3}{4}} \sum_{\substack{j_{1}+\ell=j \\
0 \leq j_{1} \leq \frac{2 r-(-1)^{r}-3}{1 \leq \ell \leq m}}} b_{1}\left(r, m, j_{1}, 2\right) d_{1}(m, \ell) \\
& +\sum_{m=0}^{\frac{2 r-(-1)^{r}-3}{4}-j} b_{1}(r, m, j, 2) d_{1}(m, 0)+b_{1}(r, 0, j, 3) \\
& -\sum_{m=0}^{\frac{2 r-(-1)^{r}-3}{4}-1} \sum_{\substack{j_{1}+\ell=j \\
0 \leq j_{1} \leq \frac{2 r-(-1)^{r}-3}{1 \leq \ell \leq m+1}-m-1}} b_{1}\left(r, m, j_{1}, 3\right) d(m, \ell), \\
& \left(0 \leq j \leq \frac{2 r-(-1)^{r}-3}{4}\right)
\end{aligned}
$$

when $r$ is even,

$$
\begin{aligned}
b_{1}(r+1, m, j, 2) & =\sum_{\ell=m-1}^{\frac{2 r-(-1)^{r}-3}{4}} b_{1}(r, \ell, j, 0) c(\ell, m), \\
& \left(1 \leq m \leq \frac{2 r-(-1)^{r}-3}{4}+1, \quad 0 \leq j \leq \frac{2 r-(-1)^{r}-3}{4}+1-m\right) ;
\end{aligned}
$$




$$
\begin{aligned}
& b_{1}(r+1,0, j, 2)=-\sum_{m=0}^{\frac{2 r-(-1)^{r}-3}{4}} \sum_{\substack{j_{1}+\ell=j \\
0 \leq j_{1} \leq \frac{2 r-(-1)^{r}-3}{1 \leq \ell \leq m+1}-m}} b_{1}\left(r, m, j_{1}, 0\right) d(m, \ell) \\
& +\sum_{m=0}^{\frac{2 r-(-1)^{r}-3}{4}-j} b_{1}(r, m, j, 1) d_{1}(m, 0)+b_{1}(r, 0, j, 0) \\
& +\sum_{m=0}^{\frac{2 r-(-1)^{r}-3}{4}} \sum_{\substack{j_{1}+\ell=j \\
0 \leq j_{1} \leq \frac{2 r-(-1)^{r}-3}{1 \leq \ell \leq m}-m}} b_{1}\left(r, m, j_{1}, 1\right) d_{1}(m, \ell), \\
& \left(0 \leq j \leq \frac{2 r-(-1)^{r}-3}{4}+1\right) \\
& b_{1}(r+1, m, j, 3)=\sum_{\ell=m}^{\frac{2 r-(-1)^{r}-3}{4}} b_{1}(r, \ell, j, 1) c_{1}(\ell, m) \text {, } \\
& \left(1 \leq m \leq \frac{2 r-(-1)^{r}-3}{4}, \quad 0 \leq j \leq \frac{2 r-(-1)^{r}-3}{4}-m\right) ; \\
& b_{1}(r+1,0, j, 3)=\sum_{\ell=0}^{\frac{2 r-(-1)^{r}-3}{4}-j} b_{1}(r, \ell, j, 1) c_{1}(\ell, 0),\left(0 \leq j \leq \frac{2 r-(-1)^{r}-3}{4}\right) \text {. }
\end{aligned}
$$

Lemma 4 ([11]). For $r, n, p \in \mathbb{N}$, we have

$$
\begin{aligned}
& H_{n}^{(p, r, 1)}= \sum_{m=0}^{\frac{2 r-(-1)^{r}-3}{4}} \frac{2 r-(-1)^{r}-3}{4}-m \\
& \sum_{j=0}\left(b_{1}(r, j, m, 0)(-1)^{n-1} H_{n}^{(p-m)}+b_{1}(r, j, m, 1) \bar{H}_{n}^{(p-m)}\right. \\
&\left.+b_{1}(r, j, m, 2) H_{n}^{(p-m)}+b_{1}(r, j, m, 3)(-1)^{n-1} \bar{H}_{n}^{(p-m)}\right) n^{j}, \\
& H_{n}^{(p, r, 3)}= \sum_{m=0}^{\frac{2 r-(-1)^{r}-3}{4}} \frac{2 r-(-1)^{r}-3}{\sum_{j=0}^{4}}-m \\
&\left.+b_{1}(r, j, m, 2) \bar{H}_{n}^{(p-m)}+b_{1}(r, j, m, m, 3)(-1)^{n-1} H_{n}^{(p-m)}\right) n^{j},
\end{aligned}
$$

where $b_{1}(r, j, m, k)(k=0,1,2,3)$ are given in Definition 2. 
Lemma $5([8,9])$. Let $\sum_{i=1}^{\infty} a_{i}$ and $\sum_{i=1}^{\infty} b_{i}$ be two series of positive terms such that $\lim _{n \rightarrow \infty} \frac{a_{i}}{b_{i}}=1$. Then if $\sum_{i=1}^{\infty} b_{i}$ is divergent, the following limit holds:

$$
\lim _{n \rightarrow \infty} \frac{\sum_{i=1}^{n} a_{i}}{\sum_{i=1}^{n} b_{i}}=1,
$$

Lemma 6 ([7, 13]). Let $p_{n, k}$ denote the $n$th squarefree number with just $k$ prime factors and $q_{n, k}$ denote the nth number with just $k$ prime factors. Then the following asymptotic relations hold:

$$
\begin{aligned}
& p_{n, k} \sim q_{n, k} \sim(k-1) ! \frac{n \log (n)}{(\log \log (n))^{k-1}}, \\
& p_{n, k}\left(\log \log \left(p_{n, k}\right)\right)^{k-1} \sim q_{n, k}\left(\log \log \left(q_{n, k}\right)\right)^{k-1} \sim(k-1) ! n \log (n) .
\end{aligned}
$$

For $k=1$, we have $p_{n} \sim n \log (n)$.

Lemma $7([13])$. For $m, n, k, x \in \mathbb{N}$, we have

$$
\begin{aligned}
& \sum_{\ell=1}^{x} \ell^{m}(\log (\ell))^{n} \sim \frac{x^{m+1}(\log (x))^{n}}{m+1}, \\
& \sum_{\ell=1}^{x} \frac{\ell^{m}(\log (\ell))^{n}}{(\log \log (\ell))^{k}} \sim \frac{x^{m+1}(\log (x))^{n}}{(m+1)(\log \log (x))^{k}} .
\end{aligned}
$$

\section{Sums over primes involving generalized alter- nating hyperharmonic numbers of type $H_{n}^{(p, r, 1)}$}

Now we will provide the asymptotic formula for the generalized alternating hyperharmonic numbers of type $H_{n}^{(p, r, 1)}$.

Lemma 8. Let $y, p \in \mathbb{N}$ with $p \geq 2$, the following asymptotic formulas hold:

$$
\begin{aligned}
& H_{n}^{(1,2 y+1,1)} \sim \frac{1}{2^{y} \cdot y !} n^{y} \log (n), \quad H_{n}^{(p, 2 y+1,1)} \sim \frac{1}{2^{y} \cdot y !} n^{y} \zeta(p), \\
& H_{n}^{(1,2 y, 1)} \sim \frac{1}{2^{y} \cdot(y-1) !} n^{y-1}(-1)^{n-1} \log (n), \\
& H_{2 n}^{(p, 2 y, 1)} \sim-\frac{1}{2 \cdot(y-1) !} n^{y-1}(\zeta(p)-\bar{\zeta}(p)), \\
& H_{2 n-1}^{(p, 2 y, 1)} \sim \frac{1}{2 \cdot(y-1) !} n^{y-1}(\zeta(p)+\bar{\zeta}(p)),
\end{aligned}
$$


where $\bar{\zeta}(s)$ denotes the well-known alternating zeta function

$$
\bar{\zeta}(s):=\sum_{n=1}^{\infty} \frac{(-1)^{n-1}}{n^{s}}=\left(1-2^{1-s}\right) \zeta(s) \quad \text { with } \quad \bar{\zeta}(1)=\log 2 .
$$

Proof. From Definition 2 and Lemma 4, we have the following identities: when $r=o d d$,

$$
H_{n}^{(p, r, 1)}=\sum_{m=0}^{\frac{2 r-(-1)^{r}-3}{4}} \sum_{j=0}^{\frac{2 r-(-1)^{r}-3}{4}-m}\left(b_{1}(r, j, m, 2) H_{n}^{(p-m)}+b_{1}(r, j, m, 3)(-1)^{n-1} \bar{H}_{n}^{(p-m)}\right) n^{j},
$$

when $r=$ even,

$$
H_{n}^{(p, r, 1)}=\sum_{m=0}^{\frac{2 r-(-1)^{r}-3}{4}} \sum_{j=0}^{\frac{2 r-(-1)^{r}-3}{4}-m}\left(b_{1}(r, j, m, 0)(-1)^{n-1} H_{n}^{(p-m)}+b_{1}(r, j, m, 1) \bar{H}_{n}^{(p-m)}\right) n^{j} .
$$

When $r=o d d$, note that $b_{1}(r, m, j, 3)=0 \quad\left(m+j=\frac{2 r-(-1)^{r}-3}{4}\right)$, we know that the main term of $H_{n}^{(p, r, 1)}$ is $b_{1}\left(r, \frac{2 r-(-1)^{r}-3}{4}, 0,2\right) H_{n}^{(p)} n^{\frac{2 r-(-1)^{r}-3}{4}}$.

When $r=$ even and $p=1$, we know that the main term of $H_{n}^{(1, r, 1)}$ is $b_{1}\left(r, \frac{2 r-(-1)^{r}-3}{4}, 0,0\right)(-1)^{n-1} H_{n} n^{\frac{2 r-(-1)^{r}-3}{4}}$.

When $r=$ even and $p \geq 2$, we know that the main term of $H_{n}^{(p, r, 1)}$ is $\left(b_{1}\left(r, \frac{2 r-(-1)^{r}-3}{4}, 0,0\right)(-1)^{n-1} H_{n}^{(p-m)}+b_{1}\left(r, \frac{2 r-(-1)^{r}-3}{4}, 0,1\right) \bar{H}_{n}^{(p)}\right) n^{\frac{2 r-(-1)^{r}-3}{4}}$.

From Definition 2 we can obtain the following recursive formulas: when $r$ is odd with $r \geq 3$,

$$
\begin{aligned}
& b_{1}\left(r+1, \frac{2(r+1)-(-1)^{r+1}-3}{4}, 0,0\right)=b_{1}\left(r, \frac{2 r-(-1)^{r}-3}{4}, 0,2\right) \frac{1}{2}, \\
& b_{1}\left(r+1, \frac{2(r+1)-(-1)^{r+1}-3}{4}, 0,1\right)=b_{1}\left(r, \frac{2 r-(-1)^{r}-3}{4}-1,0,3\right) \frac{1}{\frac{2 r-(-1)^{r}-3}{4}},
\end{aligned}
$$

when $r$ is even,

$$
\begin{aligned}
& b_{1}\left(r+1, \frac{2(r+1)-(-1)^{r+1}-3}{4}, 0,2\right)=b_{1}\left(r, \frac{2 r-(-1)^{r}-3}{4}, 0,0\right) \frac{1}{\frac{2 r-(-1)^{r}-3}{4}+1}, \\
& b_{1}\left(r+1, \frac{2(r+1)-(-1)^{r+1}-3}{4}-1,0,3\right)=b_{1}\left(r, \frac{2 r-(-1)^{r}-3}{4}-1,0,1\right) \frac{1}{2} .
\end{aligned}
$$


By using the initial values $b_{1}(1,0,0,2)=1, \quad b_{1}(1,0,0,3)=0$, and the above recursive formulas, we can give the following explicit formulas:

For $y \in \mathbb{N}$,

$$
\begin{aligned}
& b_{1}(2 y+1, y, 0,2)=\frac{1}{2^{y} \cdot y !}, \\
& b_{1}(2 y+1, y-1,0,3)=\frac{1}{2^{y+1} \cdot(y-1) !}, \\
& b_{1}(2 y, y-1,0,0)=b_{1}(2 y, y-1,0,1)=\frac{1}{2^{y} \cdot(y-1) !} .
\end{aligned}
$$

Thus we get the desired results.

Now we will prove our main theorems of this section.

Theorem 1. For $\alpha, m, q, y \in \mathbb{N}$ with $q \geq 2$, we have

$$
\begin{aligned}
& \sum_{\ell \leq x} p_{\ell}^{\alpha}\left(H_{\ell}^{(1,2 y+1,1)}\right)^{m} \sim \frac{x^{\alpha+m y+1}(\log (x))^{\alpha+m}}{\left(2^{y} \cdot y !\right)^{m}(\alpha+m y+1)}, \\
& \sum_{p_{\ell} \leq x} p_{\ell}^{\alpha}\left(H_{\ell}^{(1,2 y+1,1)}\right)^{m} \sim \frac{x^{\alpha+m y+1}}{\left(2^{y} \cdot y !\right)^{m}(\alpha+m y+1)(\log (x))^{m(y-1)+1}}, \\
& \sum_{\ell \leq x} p_{\ell}^{\alpha}\left(H_{\ell}^{(q, 2 y+1,1)}\right)^{m} \sim \frac{\zeta(q)^{m} x^{\alpha+m y+1}(\log (x))^{\alpha}}{\left(2^{y} \cdot y !\right)^{m}(\alpha+m y+1)}, \\
& \sum_{p_{\ell} \leq x} p_{\ell}^{\alpha}\left(H_{\ell}^{(q, 2 y+1,1)}\right)^{m} \sim \frac{\zeta(q)^{m} x^{\alpha+m y+1}}{\left(2^{y} \cdot y !\right)^{m}(\alpha+m y+1)(\log (x))^{m y+1}}, \\
& \sum_{\ell \leq x} p_{\ell}^{\alpha}\left((-1)^{\ell-1} H_{\ell}^{(1,2 y, 1)}\right)^{m} \sim \frac{x^{\alpha+m(y-1)+1}(\log (x))^{\alpha+m}}{\left(2^{y} \cdot(y-1) !\right)^{m}(\alpha+m(y-1)+1)}, \\
& \sum_{p_{\ell} \leq x} p_{\ell}^{\alpha}\left((-1)^{\ell-1} H_{\ell}^{(1,2 y, 1)}\right)^{m} \sim \frac{x^{\alpha+m(y-1)+1}}{\left(2^{y} \cdot(y-1) !\right)^{m}(\alpha+m(y-1)+1)(\log (x))^{m(y-2)+1}}, \\
& \sum_{\ell \leq x} p_{\ell}^{\alpha}\left(H_{2 \ell-1}^{(1,2 y, 1)}\right)^{m} \sim \sum_{\ell \leq x} p_{\ell}^{\alpha}\left(-H_{2 \ell}^{(1,2 y, 1)}\right)^{m} \sim \frac{x^{\alpha+m(y-1)+1}(\log (x))^{\alpha+m}}{(2 \cdot(y-1) !)^{m}(\alpha+m(y-1)+1)}, \\
& \sum_{p_{\ell} \leq x} p_{\ell}^{\alpha}\left(H_{2 \ell-1}^{(1,2 y, 1)}\right)^{m} \sim \sum_{p_{\ell} \leq x} p_{\ell}^{\alpha}\left(-H_{2 \ell}^{(1,2 y, 1)}\right)^{m}
\end{aligned}
$$




$$
\begin{aligned}
& \sim \frac{x^{\alpha+m(y-1)+1}}{(2 \cdot(y-1) !)^{m}(\alpha+m(y-1)+1)(\log (x))^{m(y-2)+1}}, \\
& \sum_{\ell \leq x} p_{\ell}^{\alpha}\left(H_{2 \ell-1}^{(q, 2 y, 1)}\right)^{m} \sim \frac{(\zeta(q)+\bar{\zeta}(q))^{m} x^{\alpha+m(y-1)+1}(\log (x))^{\alpha}}{(2 \cdot(y-1) !)^{m}(\alpha+m(y-1)+1)} \\
& \sum_{p_{\ell} \leq x} p_{\ell}^{\alpha}\left(H_{2 \ell-1}^{(q, 2 y, 1)}\right)^{m} \sim \frac{(\zeta(q)+\bar{\zeta}(q))^{m} x^{\alpha+m(y-1)+1}}{(2 \cdot(y-1) !)^{m}(\alpha+m(y-1)+1)(\log (x))^{m(y-1)+1}} \\
& \sum_{\ell \leq x} p_{\ell}^{\alpha}\left(-H_{2 \ell}^{(q, 2 y, 1)}\right)^{m} \sim \frac{(\zeta(q)-\bar{\zeta}(q))^{m} x^{\alpha+m(y-1)+1}(\log (x))^{\alpha}}{(2 \cdot(y-1) !)^{m}(\alpha+m(y-1)+1)} \\
& \sum_{p_{\ell} \leq x} p_{\ell}^{\alpha}\left(-H_{2 \ell}^{(q, 2 y, 1)}\right)^{m} \sim \frac{(\zeta(q)-\bar{\zeta}(q))^{m} x^{\alpha+m(y-1)+1}}{(2 \cdot(y-1) !)^{m}(\alpha+m(y-1)+1)(\log (x))^{m(y-1)+1}}
\end{aligned}
$$

Proof. By using Lemmata 5, 6, 7, and 8, we have

$$
\sum_{\ell \leq x} p_{\ell}^{\alpha}\left(H_{\ell}^{(1,2 y+1,1)}\right)^{m} \sim \sum_{\ell \leq x} \frac{\ell^{\alpha+m y}(\log (\ell))^{\alpha+m}}{\left(2^{y} \cdot y !\right)^{m}} \sim \frac{x^{\alpha+m y+1}(\log (x))^{\alpha+m}}{\left(2^{y} \cdot y !\right)^{m}(\alpha+m y+1)} .
$$

The other eleven asymptotic formulas can be proved in a similar manner.

Theorem 2. For $\alpha, m, k, q, y \in \mathbb{N}$ with $q \geq 2$, we have

$$
\begin{aligned}
& \sum_{\ell \leq x} p_{\ell, k}^{\alpha}\left(H_{\ell}^{(1,2 y+1,1)}\right)^{m} \sim \frac{((k-1) !)^{\alpha} x^{\alpha+m y+1}(\log (x))^{\alpha+m}}{\left(2^{y} \cdot y !\right)^{m}(\alpha+m y+1)(\log \log (x))^{\alpha(k-1)}}, \\
& \sum_{p_{\ell, k} \leq x} p_{\ell, k}^{\alpha}\left(H_{\ell}^{(1,2 y+1,1)}\right)^{m} \sim \frac{x^{\alpha+m y+1}(\log \log (x))^{(m y+1)(k-1)}}{\left(2^{y} \cdot y !\right)^{m}((k-1) !)^{m y+1}(\alpha+m y+1)(\log (x))^{m(y-1)+1}} \\
& \sum_{\ell \leq x} p_{\ell, k}^{\alpha}\left(H_{\ell}^{q, 2 y+1,1)}\right)^{m} \sim \frac{((k-1) !)^{\alpha} \zeta(q)^{m} x^{\alpha+m y+1}(\log (x))^{\alpha}}{\left(2^{y} \cdot y !\right)^{m}(\alpha+m y+1)(\log \log (x))^{\alpha(k-1)}}, \\
& \sum_{p_{\ell, k} \leq x} p_{\ell, k}^{\alpha}\left(H_{\ell}^{(q, 2 y+1,1)}\right)^{m} \sim \frac{\zeta(q)^{m} x^{\alpha+m y+1}(\log \log (x))^{(m y+1)(k-1)}}{\left(2^{y} \cdot y !\right)^{m}((k-1) !)^{m y+1}(\alpha+m y+1)(\log (x))^{m y+1}} \\
& \sum_{\ell \leq x} p_{\ell, k}^{\alpha}\left((-1)^{\ell-1} H_{\ell}^{1,2 y, 1)}\right)^{m} \sim \frac{((k-1) !)^{\alpha} x^{\alpha+m(y-1)+1}(\log (x))^{\alpha+m}}{\left(2^{y} \cdot(y-1) !\right)^{m}(\alpha+m(y-1)+1)(\log \log (x))^{\alpha(k-1)}} \\
& \sum_{p_{\ell, k} \leq x} p_{\ell, k}^{\alpha}\left((-1)^{\ell-1} H_{\ell}^{(1,2 y, 1)}\right)^{m} \sim \frac{x^{\alpha+m(y-1)+1}(\log \log (x))^{(m(y-1)+1)(k-1)}}{\left(2^{y} \cdot(y-1) !\right)^{m}((k-1) !)^{m(y-1)+1}} \\
& \times \frac{1}{(\alpha+m(y-1)+1)(\log (x))^{m(y-2)+1}}
\end{aligned}
$$




$$
\begin{aligned}
& \sum_{\ell \leq x} p_{\ell, k}^{\alpha}\left(H_{2 \ell-1}^{1,2 y, 1)}\right)^{m} \sim \sum_{p_{\ell, k} \leq x} p_{\ell, k}^{\alpha}\left(-H_{2 \ell}^{(1,2 y, 1)}\right)^{m} \\
& \sim \frac{((k-1) !)^{\alpha} x^{\alpha+m(y-1)+1}(\log (x))^{\alpha+m}}{(2 \cdot(y-1) !)^{m}(\alpha+m(y-1)+1)(\log \log (x))^{\alpha(k-1)}}, \\
& \sum_{p_{\ell, k} \leq x} p_{\ell, k}^{\alpha}\left(H_{2 \ell-1}^{(1,2 y, 1)}\right)^{m} \sim \sum_{p_{\ell, k} \leq x} p_{\ell, k}^{\alpha}\left(-H_{2 \ell}^{(1,2 y, 1)}\right)^{m} \\
& \sim \frac{x^{\alpha+m(y-1)+1}(\log \log (x))^{(m(y-1)+1)(k-1)}}{(2 \cdot(y-1) !)^{m}((k-1) !)^{m(y-1)+1}(\alpha+m(y-1)+1)(\log (x))^{m(y-2)+1}}, \\
& \sum_{\ell \leq x} p_{\ell, k}^{\alpha}\left(H_{2 \ell-1}^{(q, 2 y, 1)}\right)^{m} \sim \frac{((k-1) !)^{\alpha}(\zeta(q)+\bar{\zeta}(q))^{m} x^{\alpha+m(y-1)+1}(\log (x))^{\alpha}}{(2 \cdot(y-1) !)^{m}(\alpha+m(y-1)+1)(\log \log (x))^{\alpha(k-1)}}, \\
& \sum_{p_{\ell, k} \leq x} p_{\ell, k}^{\alpha}\left(H_{2 \ell-1}^{(q, 2 y, 1)}\right)^{m} \sim \frac{(\zeta(q)+\bar{\zeta}(q))^{m} x^{\alpha+m(y-1)+1}(\log \log (x))^{(m(y-1)+1)(k-1)}}{(2 \cdot(y-1) !)^{m}((k-1) !)^{m(y-1)+1}} \\
& \times \frac{1}{(\alpha+m(y-1)+1)(\log (x))^{m(y-1)+1}}, \\
& \sum_{\ell \leq x} p_{\ell, k}^{\alpha}\left(-H_{2 \ell}^{(q, 2 y, 1)}\right)^{m} \sim \frac{((k-1) !)^{\alpha}(\zeta(q)-\bar{\zeta}(q))^{m} x^{\alpha+m(y-1)+1}(\log (x))^{\alpha}}{(2 \cdot(y-1) !)^{m}(\alpha+m(y-1)+1)(\log \log (x))^{\alpha(k-1)}}, \\
& \sum_{p_{\ell, k} \leq x} p_{\ell, k}^{\alpha}\left(-H_{2 \ell}^{(q, 2 y, 1)}\right)^{m} \sim \frac{(\zeta(q)-\bar{\zeta}(q))^{m} x^{\alpha+m(y-1)+1}(\log \log (x))^{(m(y-1)+1)(k-1)}}{(2 \cdot(y-1) !)^{m}((k-1) !)^{m(y-1)+1}} \\
& \times \frac{1}{(\alpha+m(y-1)+1)(\log (x))^{m(y-1)+1}},
\end{aligned}
$$

Proof. By using Lemmata 5, 6, 7, and 8, we have

$$
\begin{aligned}
& \sum_{\ell \leq x} p_{\ell, k}^{\alpha}\left(H_{\ell}^{(1,2 y+1,1)}\right)^{m} \sim \sum_{\ell \leq x} \frac{((k-1) !)^{\alpha} \ell^{\alpha+m y}(\log (\ell))^{\alpha+m}}{\left(2^{y} \cdot y !\right)^{m}(\log \log (\ell))^{\alpha(k-1)}} \\
& \sim \frac{((k-1) !)^{\alpha} x^{\alpha+m y+1}(\log (x))^{\alpha+m}}{\left(2^{y} \cdot y !\right)^{m}(\alpha+m y+1)(\log \log (x))^{\alpha(k-1)}} .
\end{aligned}
$$

The other eleven asymptotic formulas can be proved in a similar manner. 


\section{Sums over primes involving generalized alter- nating hyperharmonic numbers of type $H_{n}^{(p, r, 2)}$}

Now we will provide the asymptotic formula for the generalized alternating hyperharmonic numbers of type $H_{n}^{(p, r, 2)}$.

Lemma 9. For $r, n, p \in \mathbb{N}$, we have

$$
H_{n}^{(p, r, 2)} \sim \frac{1}{(r-1) !} n^{r-1} \bar{\zeta}(p) .
$$

Proof. By using Lemma 3, we know that the main term of $H_{n}^{(p, r, 2)}$ is $a(r, 0, r-$ 1) $n^{r-1} \bar{H}_{n}^{(p)}$. It is known that [13] $a(r, 0, r-1)=\frac{1}{(r-1) !}$ and $\bar{H}_{n}^{(p)} \sim \bar{\zeta}(p)$. Thus we get the desired result.

Now we will prove our main theorems of this section.

Theorem 3. For $\alpha, m, q, k, r \in \mathbb{N}$, we have

$$
\begin{aligned}
& \sum_{\ell \leq x} p_{\ell}^{\alpha}\left(H_{\ell}^{(q, r, 2)}\right)^{m} \sim \frac{\bar{\zeta}(q)^{m} x^{\alpha+m(r-1)+1}(\log (x))^{\alpha}}{((r-1) !)^{m}(\alpha+m(r-1)+1)}, \\
& \sum_{p_{\ell} \leq x} p_{\ell}^{\alpha}\left(H_{\ell}^{(q, r, 2)}\right)^{m} \sim \frac{\bar{\zeta}(q)^{m} x^{\alpha+m(r-1)+1}}{((r-1) !)^{m}(\alpha+m(r-1)+1)(\log (x))^{m(r-1)+1}}, \\
& \sum_{\ell \leq x} p_{\ell, k}^{\alpha}\left(H_{\ell}^{(q, r, 2)}\right)^{m} \sim \frac{((k-1) !)^{\alpha} \bar{\zeta}(q)^{m} x^{\alpha+m(r-1)+1}(\log (x))^{\alpha}}{((r-1) !)^{m}(\alpha+m(r-1)+1)(\log \log (x))^{\alpha(k-1)}}, \\
& \sum_{p_{\ell, k} \leq x} p_{\ell, k}^{\alpha}\left(H_{\ell}^{(q, r, 2)}\right)^{m} \sim \frac{\bar{\zeta}(q)^{m} x^{\alpha+m(r-1)+1}(\log \log (x))^{(m(r-1)+1)(k-1)}}{((k-1) !)^{m(r-1)+1}((r-1) !)^{m}(\alpha+m(r-1)+1)(\log (x))^{m(r-1)+1}},
\end{aligned}
$$

Proof. By using Lemmata 5, 6, 7, and 9, we have

$$
\begin{aligned}
& \sum_{\ell \leq x} p_{\ell, k}^{\alpha}\left(H_{\ell}^{(q, r, 2)}\right)^{m} \sim \sum_{\ell \leq x} \frac{((k-1) !)^{\alpha} \bar{\zeta}(q)^{m} \ell^{\alpha+m(r-1)}(\log (\ell))^{\alpha}}{((r-1) !)^{m}(\log \log (\ell))^{\alpha(k-1)}} \\
& \sim \frac{((k-1) !)^{\alpha} \bar{\zeta}(q)^{m} x^{\alpha+m(r-1)+1}(\log (x))^{\alpha}}{((r-1) !)^{m}(\alpha+m(r-1)+1)(\log \log (x))^{\alpha(k-1)}} .
\end{aligned}
$$

The other three asymptotic formulas can be proved in a similar manner. 
Theorem 4. For $q_{1}, q_{2}, \alpha, \beta, m, k, s, n, r_{1}, r_{2} \in \mathbb{N}$ with $q_{1} \geq 2$, we have

$$
\begin{aligned}
& \sum_{\ell \leq x} p_{\ell, k}^{\alpha}\left(H_{\ell}^{\left(q_{1}, r_{1}\right)}\right)^{m}\left(h_{\ell}^{(s)}\right)^{n}\left(H_{\ell}^{\left(q_{2}, r_{2}, 2\right)}\right)^{\beta} \sim \frac{((k-1) !)^{\alpha} \zeta\left(q_{1}\right)^{m} \bar{\zeta}\left(q_{2}\right)^{\beta}(\log (x))^{\alpha+n}}{\left(\left(r_{1}-1\right) !\right)^{m}((s-1) !)^{n}\left(\left(r_{2}-1\right) !\right)^{\beta}} \\
& \times \frac{x^{\alpha+m\left(r_{1}-1\right)+n(s-1)+\beta\left(r_{2}-1\right)+1}}{\left(\alpha+m\left(r_{1}-1\right)+n(s-1)+\beta\left(r_{2}-1\right)+1\right)(\log \log (x))^{\alpha(k-1)}}, \\
& \sum_{p_{\ell, k} \leq x} p_{\ell, k}^{\alpha}\left(H_{\ell}^{\left(q_{1}, r_{1}\right)}\right)^{m}\left(h_{\ell}^{(s)}\right)^{n}\left(H_{\ell}^{\left(q_{2}, r_{2}, 2\right)}\right)^{\beta} \sim \frac{\zeta\left(q_{1}\right)^{m} \bar{\zeta}\left(q_{2}\right)^{\beta}}{\left(\alpha+m\left(r_{1}-1\right)+n(s-1)+\beta\left(r_{2}-1\right)+1\right)} \\
& \times \frac{x^{\alpha+m\left(r_{1}-1\right)+n(s-1)+\beta\left(r_{2}-1\right)+1}(\log \log (x))^{\left(m\left(r_{1}-1\right)+n(s-1)+\beta\left(r_{2}-1\right)+1\right)(k-1)}}{\left(\left(r_{1}-1\right) !\right)^{m}((s-1) !)^{n}\left(\left(r_{2}-1\right) !\right)^{\beta}((k-1) !)^{m\left(r_{1}-1\right)+n(s-1)+\beta\left(r_{2}-1\right)+1}} \\
& \times \frac{1}{(\log (x))^{m\left(r_{1}-1\right)+n(s-2)+\beta\left(r_{2}-1\right)+1}} .
\end{aligned}
$$

Proof. By using Lemmata 1, 2, 5, 6, 7, and 9, we have

$$
\begin{aligned}
& \sum_{\ell \leq x} p_{\ell, k}^{\alpha}\left(H_{\ell}^{\left(q_{1}, r_{1}\right)}\right)^{m}\left(h_{\ell}^{(s)}\right)^{n}\left(H_{\ell}^{\left(q_{2}, r_{2}, 2\right)}\right)^{\beta} \\
& \sim \sum_{\ell \leq x} \frac{((k-1) !)^{\alpha} \zeta\left(q_{1}\right)^{m} \bar{\zeta}\left(q_{2}\right)^{\beta} \ell^{\alpha+m\left(r_{1}-1\right)+n(s-1)+\beta\left(r_{2}-1\right)}(\log (\ell))^{\alpha+n}}{\left(\left(r_{1}-1\right) !\right)^{m}((s-1) !)^{n}\left(\left(r_{2}-1\right) !\right)^{\beta}(\log \log (\ell))^{\alpha(k-1)}} \\
& \sim \frac{((k-1) !)^{\alpha} \zeta\left(q_{1}\right)^{m} \bar{\zeta}\left(q_{2}\right)^{\beta}(\log (x))^{\alpha+n}}{\left(\left(r_{1}-1\right) !\right)^{m}((s-1) !)^{n}\left(\left(r_{2}-1\right) !\right)^{\beta}(\log \log (x))^{\alpha(k-1)}} \\
& \times \frac{x^{\alpha+m\left(r_{1}-1\right)+n(s-1)+\beta\left(r_{2}-1\right)+1}}{\left(\alpha+m\left(r_{1}-1\right)+n(s-1)+\beta\left(r_{2}-1\right)+1\right)} .
\end{aligned}
$$

The other asymptotic formula can be proved in a similar manner.

\section{Sums over primes involving generalized alter- nating hyperharmonic numbers of type $H_{n}^{(p, r, 3)}$}

Now we will provide the asymptotic formula for the generalized alternating hyperharmonic numbers of type $H_{n}^{(p, r, 3)}$.

Lemma 10. Let $y, p \in \mathbb{N}$, the following asymptotic formulas hold:

$$
\begin{aligned}
& H_{n}^{(p, 2 y+1,3)} \sim \frac{1}{2^{y} \cdot y !} n^{y} \bar{\zeta}(p), \\
& H_{n}^{(1,2 y, 3)} \sim \frac{1}{2^{y} \cdot(y-1) !} n^{y-1} \log (n),
\end{aligned}
$$




$$
\begin{array}{ll}
H_{2 n}^{(p, 2 y, 3)} & \sim \frac{1}{2 \cdot(y-1) !} n^{y-1}(\zeta(p)-\bar{\zeta}(p)), \quad p \geq 2, \\
H_{2 n-1}^{(p, 2 y, 3)} & \sim \frac{1}{2 \cdot(y-1) !} n^{y-1}(\zeta(p)+\bar{\zeta}(p)), \quad p \geq 2 .
\end{array}
$$

Proof. From Definition 2 and Lemma 4, we have the following identities: when $r=$ odd,

$$
H_{n}^{(p, r, 3)}=\sum_{m=0}^{\frac{2 r-(-1)^{r}-3}{4}} \sum_{j=0}^{\frac{2 r-(-1)^{r}-3}{4}-m}\left(b_{1}(r, j, m, 2) \bar{H}_{n}^{(p-m)}+b_{1}(r, j, m, 3)(-1)^{n-1} H_{n}^{(p-m)}\right) n^{j},
$$

when $r=$ even,

$$
H_{n}^{(p, r, 1)}=\sum_{m=0}^{\frac{2 r-(-1)^{r}-3}{4}} \sum_{j=0}^{\frac{2 r-(-1)^{r}-3}{4}-m}\left(b_{1}(r, j, m, 0)(-1)^{n-1} \bar{H}_{n}^{(p-m)}+b_{1}(r, j, m, 1) H_{n}^{(p-m)}\right) n^{j}
$$

When $r=o d d$, note that $b_{1}(r, m, j, 3)=0 \quad\left(m+j=\frac{2 r-(-1)^{r}-3}{4}\right)$, we know that the main term of $H_{n}^{(p, r, 3)}$ is $b_{1}\left(r, \frac{2 r-(-1)^{r}-3}{4}, 0,2\right) \bar{H}_{n}^{(p)} n^{\frac{2 r-(-1)^{r}-3}{4}}$.

When $r=$ even and $p=1$, we know that the main term of $H_{n}^{(1, r, 3)}$ is $b_{1}\left(r, \frac{2 r-(-1)^{r}-3}{4}, 0,1\right) H_{n} n^{\frac{2 r-(-1)^{r}-3}{4}}$.

When $r=$ even and $p \geq 2$, we know that the main term of $H_{n}^{(p, r, 3)}$ is $\left(b_{1}\left(r, \frac{2 r-(-1)^{r}-3}{4}, 0,0\right)(-1)^{n-1} \bar{H}_{n}^{(p-m)}+b_{1}\left(r, \frac{2 r-(-1)^{r}-3}{4}, 0,1\right) H_{n}^{(p)}\right) n^{\frac{2 r-(-1)^{r}-3}{4}}$.

From the proof of Lemma 8, we have the following explicit formulas: For $y \in \mathbb{N}$,

$$
\begin{aligned}
& b_{1}(2 y+1, y, 0,2)=\frac{1}{2^{y} \cdot y !}, \\
& b_{1}(2 y+1, y-1,0,3)=\frac{1}{2^{y+1} \cdot(y-1) !}, \\
& b_{1}(2 y, y-1,0,0)=b_{1}(2 y, y-1,0,1)=\frac{1}{2^{y} \cdot(y-1) !} .
\end{aligned}
$$

Thus we get the desired results.

Now we will prove our main theorems of this section. 
Theorem 5. For $\alpha, m, p, q, y \in \mathbb{N}$ with $q \geq 2$, we have

$$
\begin{aligned}
\sum_{\ell \leq x} p_{\ell}^{\alpha}\left(H_{\ell}^{(p, 2 y+1,3)}\right)^{m} & \sim \frac{\bar{\zeta}(p)^{m} x^{\alpha+m y+1}(\log (x))^{\alpha}}{\left(2^{y} \cdot y !\right)^{m}(\alpha+m y+1)} \\
\sum_{p_{\ell} \leq x} p_{\ell}^{\alpha}\left(H_{\ell}^{(p, 2 y+1,3)}\right)^{m} & \sim \frac{\bar{\zeta}(p)^{m} x^{\alpha+m y+1}}{\left(2^{y} \cdot y !\right)^{m}(\alpha+m y+1)(\log (x))^{m y+1}}, \\
\sum_{\ell \leq x} p_{\ell}^{\alpha}\left(H_{\ell}^{(1,2 y, 3)}\right)^{m} & \sim \frac{x^{\alpha+m(y-1)+1}(\log (x))^{\alpha+m}}{\left(2^{y} \cdot(y-1) !\right)^{m}(\alpha+m(y-1)+1)} \\
\sum_{p_{\ell} \leq x} p_{\ell}^{\alpha}\left(H_{\ell}^{(1,2 y, 3)}\right)^{m} & \sim \frac{x^{\alpha+m(y-1)+1}}{\left(2^{y} \cdot(y-1) !\right)^{m}(\alpha+m(y-1)+1)(\log (x))^{m(y-2)+1}}, \\
\sum_{\ell \leq x} p_{\ell}^{\alpha}\left(H_{2 \ell-1}^{(q, 2 y, 3)}\right)^{m} & \sim \frac{(\zeta(q)+\bar{\zeta}(q))^{m} x^{\alpha+m(y-1)+1}(\log (x))^{\alpha}}{(2 \cdot(y-1) !)^{m}(\alpha+m(y-1)+1)} \\
\sum_{p_{\ell} \leq x} p_{\ell}^{\alpha}\left(H_{2 \ell-1}^{(q, 2 y, 3)}\right)^{m} & \sim \frac{(\zeta(q)+\bar{\zeta}(q))^{m} x^{\alpha+m(y-1)+1}}{(2 \cdot(y-1) !)^{m}(\alpha+m(y-1)+1)(\log (x))^{m(y-1)+1}} \\
\sum_{\ell \leq x} p_{\ell}^{\alpha}\left(H_{2 \ell}^{(q, 2 y, 3)}\right)^{m} & \sim \frac{(\zeta(q)-\bar{\zeta}(q))^{m} x^{\alpha+m(y-1)+1}(\log (x))^{\alpha}}{(2 \cdot(y-1) !)^{m}(\alpha+m(y-1)+1)} \\
\sum_{p_{\ell} \leq x} p_{\ell}^{\alpha}\left(H_{2 \ell}^{(q, 2 y, 3)}\right)^{m} & \sim \frac{(\zeta(q)-\bar{\zeta}(q))^{m} x^{\alpha+m(y-1)+1}}{(2 \cdot(y-1) !)^{m}(\alpha+m(y-1)+1)(\log (x))^{m(y-1)+1}}
\end{aligned}
$$

Proof. By using Lemmata 5, 6, 7, and 10, we have

$$
\sum_{\ell \leq x} p_{\ell}^{\alpha}\left(H_{\ell}^{(p, 2 y+1,3)}\right)^{m} \sim \sum_{\ell \leq x} \frac{\bar{\zeta}(p)^{m} \ell^{\alpha+m y}(\log (\ell))^{\alpha}}{\left(2^{y} \cdot y !\right)^{m}} \sim \frac{\bar{\zeta}(p)^{m} x^{\alpha+m y+1}(\log (x))^{\alpha}}{\left(2^{y} \cdot y !\right)^{m}(\alpha+m y+1)}
$$

The other seven asymptotic formulas can be proved in a similar manner.

Theorem 6. For $\alpha, m, k, p, q, y \in \mathbb{N}$ with $q \geq 2$, we have

$$
\begin{aligned}
& \sum_{\ell \leq x} p_{\ell, k}^{\alpha}\left(H_{\ell}^{p, 2 y+1,1)}\right)^{m} \sim \frac{((k-1) !)^{\alpha} \bar{\zeta}(p)^{m} x^{\alpha+m y+1}(\log (x))^{\alpha}}{\left(2^{y} \cdot y !\right)^{m}(\alpha+m y+1)(\log \log (x))^{\alpha(k-1)}}, \\
& \sum_{p_{\ell, k} \leq x} p_{\ell, k}^{\alpha}\left(H_{\ell}^{(p, 2 y+1,1)}\right)^{m} \sim \frac{\bar{\zeta}(p)^{m} x^{\alpha+m y+1}(\log \log (x))^{(m y+1)(k-1)}}{\left(2^{y} \cdot y !\right)^{m}((k-1) !)^{m y+1}(\alpha+m y+1)(\log (x))^{m y+1}}, \\
& \sum_{\ell \leq x} p_{\ell, k}^{\alpha}\left(H_{\ell}^{1,2 y, 3)}\right)^{m} \sim \frac{((k-1) !)^{\alpha} x^{\alpha+m(y-1)+1}(\log (x))^{\alpha+m}}{\left(2^{y} \cdot(y-1) !\right)^{m}(\alpha+m(y-1)+1)(\log \log (x))^{\alpha(k-1)}},
\end{aligned}
$$




$$
\begin{aligned}
& \sum_{p_{\ell, k} \leq x} p_{\ell, k}^{\alpha}\left(H_{\ell}^{(1,2 y, 3)}\right)^{m} \sim \frac{x^{\alpha+m(y-1)+1}(\log \log (x))^{(m(y-1)+1)(k-1)}}{\left(2^{y} \cdot(y-1) !\right)^{m}((k-1) !)^{m(y-1)+1}} \\
& \times \frac{1}{(\alpha+m(y-1)+1)(\log (x))^{m(y-2)+1}}, \\
& \sum_{\ell \leq x} p_{\ell, k}^{\alpha}\left(H_{2 \ell-1}^{(q, 2 y, 3)}\right)^{m} \sim \frac{((k-1) !)^{\alpha}(\zeta(q)+\bar{\zeta}(q))^{m} x^{\alpha+m(y-1)+1}(\log (x))^{\alpha}}{(2 \cdot(y-1) !)^{m}(\alpha+m(y-1)+1)(\log \log (x))^{\alpha(k-1)}}, \\
& \sum_{p_{\ell, k} \leq x} p_{\ell, k}^{\alpha}\left(H_{2 \ell-1}^{(q, 2 y, 3)}\right)^{m} \sim \frac{(\zeta(q)+\bar{\zeta}(q))^{m} x^{\alpha+m(y-1)+1}(\log \log (x))^{(m(y-1)+1)(k-1)}}{(2 \cdot(y-1) !)^{m}((k-1) !)^{m(y-1)+1}} \\
& \sum_{\ell \leq x} p_{\ell, k}^{\alpha}\left(H_{2 \ell}^{(q, 2 y, 3)}\right)^{m} \sim \frac{((k-1) !)^{\alpha}(\zeta(q)-\bar{\zeta}(q))^{m} x^{\alpha+m(y-1)+1}(\log (x))^{\alpha}}{(\alpha+m(y-1)},(y-1) !)^{m}(\alpha+m(y-1)+1)(\log \log (x))^{\alpha(k-1)} \\
& \sum_{p_{\ell, k \leq x} p_{\ell, k}^{\alpha}\left(H_{2 \ell}^{(q, 2 y, 3)}\right)^{m}} \sim \frac{(\zeta(q)-\bar{\zeta}(q))^{m} x^{\alpha+m(y-1)+1}(\log \log (x))^{(m(y-1)+1)(k-1)}}{(2 \cdot(y-1) !)^{m}((k-1) !)^{m(y-1)+1}} \\
& \times \frac{1}{(\alpha+m(y-1)+1)(\log (x))^{m(y-1)+1}},
\end{aligned}
$$

Proof. By using Lemmata 5, 6, 7, and 10, we have

$$
\begin{aligned}
& \sum_{\ell \leq x} p_{\ell, k}^{\alpha}\left(H_{\ell}^{(p, 2 y+1,3)}\right)^{m} \sim \sum_{\ell \leq x} \frac{((k-1) !)^{\alpha} \bar{\zeta}(p)^{m} \ell^{\alpha+m y}(\log (\ell))^{\alpha}}{\left(2^{y} \cdot y !\right)^{m}(\log \log (\ell))^{\alpha(k-1)}} \\
& \sim \frac{((k-1) !)^{\alpha} \bar{\zeta}(p)^{m} x^{\alpha+m y+1}(\log (x))^{\alpha}}{\left(2^{y} \cdot y !\right)^{m}(\alpha+m y+1)(\log \log (x))^{\alpha(k-1)}} .
\end{aligned}
$$

The other seven asymptotic formulas can be proved in a similar manner.

\section{Data availability}

The datasets generated during and/or analysed during the current study are available from the corresponding author on reasonable request.

\section{References}

[1] J.H. Conway, R.K. Guy, The Book of Numbers, Springer, New York, 1996. 
[2] C. de la Vallée Poussin, Recherches analytiques sur la théorie des nombres premiers. Ann. Soc. Sci. Bruxelles. 20 (1896), 183-256.

[3] A. Dil, I. Mező, M. Cenkci. Evaluation of Euler-like sums via Hurwitz zeta values. Turkish J. Math. 41 (2017), no. 6, 1640-1655.

[4] J. Gerard, L.C. Washington, Sums of powers of primes. Ramanujan J. 45 (2018), no. 1, 171-180.

[5] A. Granville, Analytic Number Theory Revealed: The Distribution of Prime Pumbers. Book draft, unpublished.

[6] J. Hadamard, Sur la distribution des zéros de la fonction $\zeta(s)$ et ses conséquences arithmétiques. Bull. Soc. Math. France 24 (1896), 199220 .

[7] G.H. Hardy, E.M. Wright, An Introduction to the Theory of Numbers. 6th edn (Oxford University Press, Oxford, 2008).

[8] R. Jakimczuk, A note on sums of powers which have a fixed number of prime factors. JIPAM. J. Inequal. Pure Appl. Math. 6 (2005), no. 2, Article 31, 4 pp.

[9] R. Jakimczuk, Functions of slow increase and integer sequences. J. Integer Seq. 13 (2010), no. 1, Article 10.1.1, 14 pp.

[10] E. Landau, Sur quelques problèmes relatifs à la distribution des nombres premiers. Bull. Soc. Math. France 28 (1900), 25-38.

[11] R. Li, Euler sums of generalized alternating hyperharmonic numbers. Rocky Mountain J. Math. 51 (2021), no. 4, 1299-1313.

[12] R. Li, Euler sums of generalized hyperharmonic numbers. Submitted, arXiv:2103.10622.

[13] R. Li, Sums over primes. Integers 21 (2021), Paper No. A94, 7 pp.

[14] I. Mező, A. Dil, Hyperharmonic series involving Hurwitz zeta function. J. Number Theory 130 (2010), 360-369.

[15] N. Ömür, S. Koparal, On the matrices with the generalized hyperharmonic numbers of order $r$. Asian-Eur. J. Math. 11 (2018), no. 3, 1850045, 9 pp.

[16] T. Šalát, Š. Znám, On sums of the prime powers. Acta Fac. Rerum Natur. Univ. Comenian. Math. 21 (1968), 21-24 (1969). 